\title{
Does Intravenous Acetaminophen Improve Postoperative Pain Control after Laparoscopic Appendectomy for Perforated Appendicitis? A Prospective Randomized Trial
}

\author{
Richard Sola Jr. ${ }^{1} \quad$ Amita A. Desai ${ }^{1} \quad$ Katherine W. Gonzalez ${ }^{1} \quad$ Nichole M. Doyle $^{2}$ Katrina L. Weaver ${ }^{1}$ \\ Ashwini S. Poola ${ }^{1}$ Jason D. Fraser ${ }^{1}$ Shawn D. St. Peter ${ }^{1}$ Daniel L. Millspaugh ${ }^{2}$ \\ ${ }^{1}$ Department of Surgery, Children's Mercy Hospitals and Clinics, \\ Kansas City, Missouri, United States \\ 2 Department of Anesthesiology, Children's Mercy Hospitals and \\ Clinics, Kansas City, Missouri, United States \\ Address for correspondence Daniel L. Millspaugh, MD, Department \\ of Anesthesiology, Children's Mercy Hospitals and Clinics, 2401 \\ Gillham Road, Kansas City, MO 64108-4619, United States \\ (e-mail: dmillspaugh@cmh.edu).
}

Eur J Pediatr Surg 2019;29:159-165.

\begin{abstract}
Keywords

- appendicitis

- acetaminophen

- children

- appendectomy

- pain

Introduction The recent increased awareness of the dangers of opioids in the United States has highlighted the need to minimize narcotics and identify nonopioid options for pain control after surgery. With evidence suggesting that intravenous acetaminophen (IVA) can be an opioid sparing option, we conducted a prospective, randomized trial that evaluated the effect of IVA on the postoperative pain course of children with perforated appendicitis.

Materials and Methods After IRB approval, children with perforated appendicitis were randomized to receive postoperative IVA with the standard patient/nursecontrolled analgesia (PCA) or to receive the PCA alone. All patients were treated according to an evidence-based treatment protocol. The primary outcome was duration of time on PCA.

Results Eighty-two patients were analyzed from $7 / 14$ to $11 / 15$. There was no statistically significant difference in the time to transition from the PCA to oral pain medications for children given IVA compared with children not receiving IVA (76.4 \pm 32.5 versus $86.7 \pm 49.3$ hours; $p=0.73$ ). Children in the IVA group had no statistically significant difference in intravenous narcotics delivered and pain scores compared with the non-IVA group. There was no significant difference in the amount of oral narcotics between both groups $(2.8 \pm 2.4$ versus $2.9 \pm 2.5 ; p=0.88)$. Patients who received IVA had higher medication charges $(\$ 3752.7 \pm 1618.3$ vs. $\$ 1198.19 \pm 521.51 ; p<0.01)$, but not total hospital charges $(\$ 53842.0 \pm 19409.2$ vs. $\$ 50501.03 \pm 16223.32 ; p=0.76)$.

Conclusion Children given IVA showed no difference in the transition time off the PCA and to oral pain medications after laparoscopic appendectomy for perforated appendicitis.
\end{abstract}

received

July 18, 2017

accepted after revision

November 14, 2017

published online

January 2, 2018 (c) 2019 Georg Thieme Verlag KG

Stuttgart · New York
DOI https://doi.org/

10.1055/s-0037-1615276.

ISSN 0939-7248. 


\section{Introduction}

Acute appendicitis remains the most common inpatient abdominal pathology and an appendectomy is the most common abdominal surgery in children. ${ }^{1}$ Approximately 25 to $38 \%$ are found to have perforated appendicitis. ${ }^{2-4}$ Those with perforated appendicitis have higher morbidity rates especially higher rates of postoperative infection and longer hospital stays compared with those undergoing appendectomy for uncomplicated appendicitis. ${ }^{4-6}$

Managing postoperative pain with perforated appendicitis can be a challenge. Evidence has shown that $32 \%$ have significant pain on the day of surgery and $18 \%$ will continue to have significant pain over the course of their hospital stay. ${ }^{7}$ Narcotics come with known adverse effects and risks. ${ }^{8-11}$ Numerous publications have investigated means to minimize narcotic burdens for children after surgery. ${ }^{12-17}$ We know that a previous retrospective review on the use of a multimodal analgesic regimen after laparoscopic appendectomy documented a reduction in postoperative pain. ${ }^{18}$ Yet, there is no prospective randomized trial evaluating multimodal analgesic therapy for children after appendectomy.

The objective of this study is to delineate the impact of intravenous acetaminophen (IVA) on the postoperative course of children who undergo laparoscopic appendectomy for perforated appendicitis. Our primary outcome was time to transition off patient/nurse-controlled analgesia (PCA) to oral pain medications. Our main secondary outcomes include amount of narcotic medication given and pain scores.

\section{Materials and Methods}

Approval was obtained from the Institutional Review Board (IRB\# 13100328) prior to enrolling patients in this study. Children were subsequently enrolled after obtaining informed permission from the legal guardian. Patient's signed assent was waived as all patients included were in pain and under the influence of pain medications due to perforated appendicitis. The enrollment process occurred after confirmation of perforated appendicitis either preoperatively by imaging or intraoperatively. The permission forms and consent process were audited by the IRB on a continuing basis. The study was registered with clinicaltrials.gov with the initial release date 1/18/16 (NCT02881996). There was no funding source.

\section{Participants}

The study population consisted of children from 2 to 17 years old that were found to have perforated appendicitis. Those found to have nonperforated appendicitis and/or a normal appendix at the time of the operation were excluded. Those with history of chronic pain, known underlying liver disorders, and allergy to pain medication in protocol and nonEnglish speaking patients were also excluded.

\section{Sample Size}

Power calculations were based on the known average length of time on PCA of 3.5 days with a standard deviation of 1.5 with goal time off PCA of 2.5 days in the treatment group.
These data points were gathered from our previous randomized trial. ${ }^{5}$ Using these numbers with an $\alpha=0.05$ and power of 0.9 , we calculated a sample size of 80 patients with 40 patients in each study arm. We intended to recruit 100 patients and have 80 randomized.

\section{Assignment}

A computer-generated individual unit of randomization was utilized in a nonstratified sequence in blocks of four. Consent was obtained by pediatric surgery fellows and pediatric surgery research fellows. After consent for study enrollment was obtained, a sequentially numbered opaque envelope was accessed to obtain the next allotment. The attending surgeons and anesthesiologists did not obtain consent and were blind to the allotment throughout the enrollment process. All data were analyzed on an intention-to-treat basis, and patients remained in their assigned group. The rounding teams were not blinded to randomization as the both the surgical and anesthesiology teams participated in the pain management during the course of hospitalization.

\section{Protocol}

Preoperatively, patients were given as needed intravenous fentanyl, morphine, or hydromorphone boluses for pain control. A balanced sevoflurane-based general anesthetic with tracheal intubation was utilized. Induction was performed with weight appropriate doses of propofol, rocuronium, fentanyl, and lidocaine. Additional opioids were given intraoperatively based on response to surgical stimulation, and dexmedetomidine was commonly given to mitigate potential emergence delirium or agitation. Intraoperative anesthetic regimen included wound infiltration with local anesthetic.

Postoperatively, all patients were placed on a morphine or hydromorphone PCA pump, which was managed by the anesthesia department. Morphine was dosed $20 \mathrm{mcg} / \mathrm{kg}$ with 8 -minute lockout along with a $20 \mathrm{mcg} / \mathrm{kg} / \mathrm{h}$ continuous infusion. Hydromorphone was dosed at $4 \mathrm{mcg} / \mathrm{kg}$ with 8-minute lockout along with a $4 \mathrm{mcg} / \mathrm{kg} / \mathrm{h}$ continuous infusion. All continuous infusions were left for at least 24 hours and then were switched to nightly infusions (10 p.m.-8 a.m.) at the discretion of the anesthesiologist. As per our existing protocol, IV ketorolac ( $0.5 \mathrm{mg} / \mathrm{kg}$ every 6 hours) was provided for 5 days. Ketorolac was held if urine output was below $0.5 \mathrm{cc} / \mathrm{h}$.

Those patients in the treatment arm received their first dose of IVA 3 hours after their initial dose of ketorolac. IVA was dosed at $10 \mathrm{mg} / \mathrm{kg}$ and scheduled every 6 hours for a total of 3 days staggered in between doses of ketorolac. If the PCA is discontinued prior to 3 days, the IVA was also discontinued as patients were then transitioned to oral analgesics.

Patients in the control arm were managed with scheduled ketorolac and PCA pump without IVA. Patients in the control group only received by mouth or per rectum acetaminophen as needed for treatment of fevers. PCA pumps were discontinued once the patient had a return of bowel function and were tolerating oral intake. The surgical and anesthesia team collaborated and mutually decided when to discontinue the PCA. 
Patients were transitioned to our standard postoperative pain regimen of $0.1 \mathrm{mg} / \mathrm{kg}$ oral oxycodone/acetaminophen $5 / 325$ every 4 hours as needed.

The patient's pain control was measured by utilizing the Visual Analogue Scale (VAS) twice daily. For patients less than 7 years old, the validated Wong-Baker FACES score was used. The scores were obtained by the pediatric surgery fellow and/or pediatric surgery research fellow, who were appropriately trained in administering and interpreting these scoring tools.

\section{Data Collection}

A single individual who had no role in the clinical care, collected all data prospectively. Demographics collected included age, weight, height, body mass index (BMI), and gender. Prehospital characteristics, operative characteristics, complications, radiographic findings, and hospital charges were also recorded. Hospital outcomes include length of stay, time to return of bowel function, time to completion of oral medications, time to initiation of diet, narcotic associated adverse effects (nausea, emesis, respiratory depression), and overall hospital cost. Total PCA narcotic doses were converted to morphine equivalent dose.

\section{Statistics}

All data were analyzed on intention-to-treat basis. Descriptive statistics including means, standard deviations, medians, interquartile ranges, counts, and percentages were analyzed. Pearson's chi-square and Fisher's exact tests were used for categorical variables. Continuous variables were compared using Student's two-sample t-test and Mann-Whitney $U$-test. Hodges-Lehmann 95\% confidence intervals (CIs) for location shift were used for those variables with $p$-values determined by Mann-Whitney $U$-Test. Statistical significance was set at $p<0.05$, and all reported $p$-values are two-tailed. Statistical analysis was performed using IBM SPSS Statistics (version 23, IBM Corp., Armonk, New York, United States).

\section{Results}

From July, 2014 to November, 2015, 90 children were randomized to either receive IVA or no IVA ( $\sim$ Fig. 1 ). During this time frame, 99 patients were identified to have perforated appendicitis. All families were approached for enrollment in the study. Nine declined to participate. Forty-five were allocated to the IVA arm and 45 to the no IVA arm. Six patients were excluded from the IVA arm. One patient did not have perforated appendicitis, five patients deviated from protocol. Two patients from the non-IVA arm were excluded due to protocol deviation. A total of 82 patients ( 39 in the IVA arm and 43 in the non-IVA arm) were analyzed.

\section{Pain Characteristics}

There was no statistically significant difference in the time on the PCA ( $p=0.27 ; 95 \% \mathrm{CI}:-28.9$ to 8.2$)$ and the time to transition from the PCA to oral pain medications for children given IVA compared with children not receiving IVA ( $p=0.27$; $95 \%$ CI: -29.7 to 8.4). Both groups showed no difference in the amount of intravenous narcotic pain medicine given and pain scores. There was no difference in the amount of oral narcotics between both groups ( $p=0.76$; $95 \% \mathrm{CI}:-1.0$ to 1.0 ). The PCA alone group received significantly more oral acetaminophen compared with the IVA group ( $p=0.01 ; 95 \% \mathrm{CI}:-2.0$ to 0 ). Total pain medication charges were significantly higher for the IVA group compared with the PCA alone group ( $p<0.01 ; 95 \%$ CI: 1914.4-2514.8) (-Table 1).

\section{Patient Characteristics}

Males accounted for 64 and $70 \%$ in each study arm. They were similar in age and BMI. There was no significant statistical difference in the admission white blood cell count, and duration of symptoms (-Table 2 ).

\section{Postoperative Outcomes}

Those given IVA had a statistically significant longer operative time compared with patients with a PCA alone $(p<0.01$;

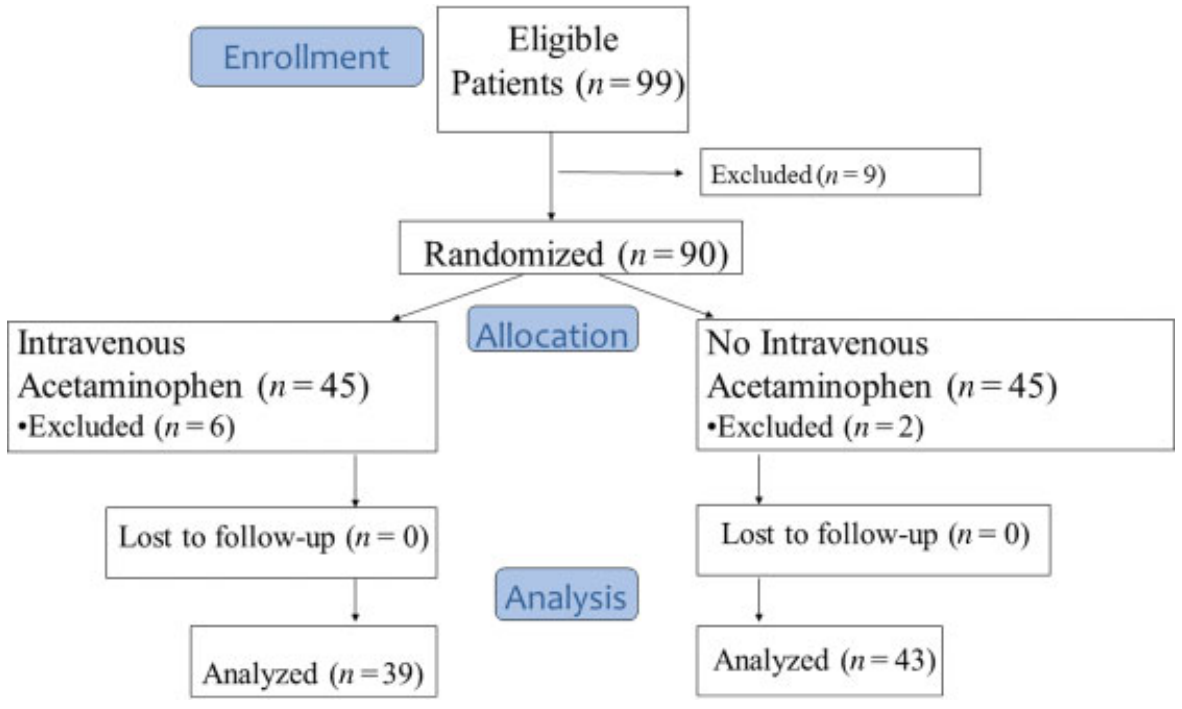

Fig. 1 CONSORT (Consolidated Standards of Reporting Trials) flow diagram. 
Table 1 Pain characteristics

\begin{tabular}{|c|c|c|c|c|}
\hline & $\begin{array}{l}\text { IVA and PCA } \\
(n=39)\end{array}$ & $\begin{array}{l}\text { PCA alone } \\
(n=43)\end{array}$ & $p$-Value & $95 \% \mathrm{Cl}$ \\
\hline Time on PCA (h) & $76.4 \pm 32.5$ & $86.7 \pm 49.3$ & 0.27 & -28.9 to 8.2 \\
\hline \multicolumn{5}{|l|}{ PCA delivered $(\mathrm{mg} / \mathrm{kg} / \text { day })^{\mathrm{a}, \mathrm{b}}$} \\
\hline POD \#1 & $2.0(1.0,4.5)$ & $1.9(0.8,3.5)$ & 0.35 & -0.4 to 1.3 \\
\hline POD \#2 & $9.5(5.0,14.9)$ & $8.4(4.0,16.7)$ & 0.64 & -2.4 to 3.4 \\
\hline POD \#3 & $9.1(3.8,15.5)$ & $10.5(3.1,19.5)$ & 0.63 & -4.6 to 2.7 \\
\hline POD \#4 & $6.9(1.4,13.6)$ & $10.9(5.3,18.3)$ & 0.06 & -8.9 to 0.2 \\
\hline POD \#5 & $5.9(1.5,11.4)$ & $8.6(7.3,24.5)$ & 0.20 & -8.7 to 2.0 \\
\hline \multicolumn{5}{|l|}{ VAS score } \\
\hline POD \#1 a.m. & $2.5 \pm 1.9$ & $2.3 \pm 2.0$ & 0.64 & \\
\hline POD \#1 p.m. & $3.2 \pm 2.3$ & $2.6 \pm 2.5$ & 0.30 & \\
\hline POD \#2 a.m. & $2.1 \pm 2.1$ & $2.8 \pm 1.9$ & 0.13 & \\
\hline POD \#2 p.m. & $2.4 \pm 2.1$ & $2.4 \pm 2.0$ & 0.95 & \\
\hline POD \#3 a.m. & $2.0 \pm 2.2$ & $2.7 \pm 2.5$ & 0.30 & \\
\hline POD \#3 p.m. & $2.3 \pm 2.1$ & $2.3 \pm 1.9$ & 0.93 & \\
\hline Transition to oral pain medications (h) & $78.6 \pm 33.6$ & $89.2 \pm 49.7$ & 0.27 & -29.7 to 8.4 \\
\hline $\begin{array}{l}\text { Total no. of oral } \\
\text { narcotics (doses) }\end{array}$ & $2(1,3)$ & $2(1,4)$ & 0.76 & -1.0 to 1.0 \\
\hline $\begin{array}{l}\text { Total no. of oral } \\
\text { acetaminophen (doses) }^{\mathrm{b}}\end{array}$ & $0.5(0,2)$ & $2(0,5)$ & 0.01 & -2.0 to 0 \\
\hline Total pain medication charges ${ }^{b}$ & $\begin{array}{l}\$ 3,395.67(2,842.23 \\
3,964.51)\end{array}$ & $\begin{array}{l}\$ 1,127.03(825.05 \\
1,481.32)\end{array}$ & $<0.01$ & $1914.4-2514.8$ \\
\hline
\end{tabular}

Abbreviations: Cl, confidence interval; IQR, interquartile range; IVA, intravenous acetaminophen; PCA, patient-controlled analgesia; POD, postoperative day; VAS, visual analog scale.

${ }^{a}$ Reported as morphine equivalents.

${ }^{b}$ Reported as median (IQR).

Table 2 Patient characteristics

\begin{tabular}{|c|c|c|c|c|}
\hline & $\begin{array}{l}\text { IVA and PCA } \\
(n=39)\end{array}$ & $\begin{array}{l}\text { PCA alone } \\
(n=43)\end{array}$ & $p$-Value & $95 \% \mathrm{Cl}$ \\
\hline \multicolumn{5}{|l|}{ Gender } \\
\hline Male, no. (\%) & $25(64)$ & $30(70)$ & \multirow[t]{2}{*}{0.59} & \\
\hline Female, no. (\%) & $14(36)$ & $13(30)$ & & \\
\hline Age (y) & $10.9 \pm 4.0$ & $9.6 \pm 4.0$ & 0.14 & -0.43 to 3.09 \\
\hline BMI $\left(\mathrm{kg} / \mathrm{m}^{2}\right)$ & $19.3 \pm 5.5$ & $18.7 \pm 5.4$ & 0.61 & -1.78 to 3.02 \\
\hline Admission WBC count $\left(10^{3} \mathrm{mcL}\right)$ & $18.0 \pm 4.2$ & $18.6 \pm 6.9$ & 0.64 & -3.24 to 2.00 \\
\hline Duration of symptoms (d) & $3.9 \pm 2.7$ & $2.9 \pm 1.8$ & 0.07 & -0.06 to 1.95 \\
\hline
\end{tabular}

Abbreviations: BMI, body mass index; $\mathrm{Cl}$, confidence interval; IVA, intravenous acetaminophen; PCA, patient-controlled analgesia; WBC, white blood cell.

95\% CI: 3.96-18.40). During the hospital stay, there was no significant statistical difference in temperature on POD\#1; however, children with PCA alone had significantly higher temperatures on POD\#2 to 5. There was no statistical significant difference in time to initial enteral feeding $(p=0.28$;
95\% CI: -5.27 to 17.69$)$, time to return of bowel function ( $p=0.49 ; 95 \% \mathrm{CI}:-17.45$ to 8.37 ), and number of antiemetic medications given ( $p=0.95 ; 95 \% \mathrm{CI}:-1.0$ to 1.0 ). There was no statistically significant difference with the incidence of postoperative abscess $(p=0.55)$ and the time to abscess 
Table 3 Postoperative outcomes

\begin{tabular}{|c|c|c|c|c|}
\hline & $\begin{array}{l}\text { IVA and PCA } \\
(n=39)\end{array}$ & $\begin{array}{l}\text { PCA alone } \\
(n=43)\end{array}$ & p-Value & $95 \% \mathrm{Cl}$ \\
\hline Operative time (min) & $45.9 \pm 21.1$ & $34.8 \pm 10.5$ & $<0.01$ & $3.96-18.40$ \\
\hline \multicolumn{5}{|l|}{ Maximum temperature $\left({ }^{\circ} \mathrm{C}\right)^{\mathrm{a}}$} \\
\hline POD \#1 & $38.0(37.6,39.2)$ & $38.6(37.9,39.5)$ & 0.11 & -0.8 to 0.1 \\
\hline POD \#2 & $37.8(37.4,38.8)$ & $38.7(37.8,39.3)$ & 0.01 & -1.1 to $(-0.1)$ \\
\hline POD \#3 & $37.4(37.1,37.9)$ & $37.8(37.3,38.7)$ & 0.02 & -0.7 to $(-0.1)$ \\
\hline POD \#4 & $37.5(37.2,37.8)$ & $37.9(37.5,38.4)$ & 0.03 & -0.6 to 0.0 \\
\hline POD \#5 & $37.3(37.1,37.5)$ & $37.9(37.5,38.2)$ & $<0.01$ & -0.8 to $(-0.3)$ \\
\hline Time to initial enteral feeding (h) & $42.9 \pm 32.0$ & $36.6 \pm 19.2$ & 0.28 & -5.27 to 17.69 \\
\hline Time to return of bowel function (h) & $30.6 \pm 29.8$ & $35.1 \pm 29.8$ & 0.49 & -17.45 to 8.37 \\
\hline Total \# of antiemetic medication given (doses) ${ }^{a}$ & $1.5(0,4.5)$ & $1(0,4)$ & 0.95 & -1.0 to 1.0 \\
\hline Abscess formation, no. (\%) & $7(18)$ & $10(23)$ & 0.55 & \\
\hline Time to abscess formation (d) & $7.2 \pm 1.6$ & $8.3 \pm 2.1$ & 0.28 & -3.29 to 18.41 \\
\hline Postoperative length of stay $(d)^{a}$ & $3.9(3.1,5.9)$ & $4.5(3.1,6.1)$ & 0.35 & -1.2 to 0.3 \\
\hline Total hospital charges $^{a}$ & $\begin{array}{l}\$ 46,947.13 \\
(41,336.43 \\
62,803.70)\end{array}$ & $\begin{array}{l}\$ 46,593 \\
(39,230.48 \\
55,614.07)\end{array}$ & 0.42 & $\begin{array}{l}-3,762.19 \text { to } \\
9,263.39\end{array}$ \\
\hline
\end{tabular}

Abbreviations: Cl, confidence interval; IQR, interquartile range; IVA, intravenous acetaminophen; PCA, patient-controlled analgesia; $\mathrm{POD}$, postoperative day.

${ }^{\mathrm{a}}$ Reported as median (IQR).

formation ( $p=0.28 ; 95 \% \mathrm{CI}$ : -3.29 to 18.41$)$. The non-IVA group did stay close to a half day longer postoperatively compared with the IVA group; however, this was not a statistically significant difference $(p=0.35 ; 95 \% \mathrm{CI}:-1.2$ to 0.3 ). There was no difference in the hospital charges between the two groups ( $p=0.42 ; 95 \% \mathrm{CI}:-3762.19$ to 9263.39) (-Table 3).

\section{Discussion}

This is the first randomized control trial evaluating the use of IVA after laparoscopic appendectomy for perforated appendicitis. In our study, there was no statistically significant difference in the time off the PCA, and transition time to oral pain medications. The IVA was not effective in reducing parenteral and oral narcotic requirements during their hospital stay and patients reported similar pain scores. There was an increased medication cost for the IVA group; however, there was no statistically significant difference in overall hospital costs between the groups due to the large denominator.

Children with generalized peritonitis due to their appendicitis have been shown to have more pain, consume more opioids, and have an increased PCA demand in comparison to those without peritonitis. ${ }^{18}$ The increase in opioid use and abuse in the United States along with lowering the incidence of opioid-related adverse effects has led to an emphasis in optimizing nonnarcotic pain management especially after surgery. ${ }^{19-21}$ In the adult population, IVA has been shown to reduce the need for opioids, lowering hospital costs, and decreasing postoperative pain visits to the emergency department. ${ }^{22-24}$ In children, recent systemic reviews have shown an opioid sparing effect for IVA after major abdominal surgery. ${ }^{25,26}$ While there has been randomized trials for postoperative pain control after open appendectomy, there is limited evidence identifying a postoperative pain regimen for laparoscopic appendectomy that can minimize the use of opioids. ${ }^{16,18,27}$ In fact, PCAs are currently still being used for postoperative pain management after appendectomies, though current evidence suggests that the need for a PCA may be declining. ${ }^{28,29}$

We felt that utilizing PCAs was crucial for providing a background environment to study the impact of scheduled IVA because each patient would have similar background demands and allow for sensitive detection of a difference created by a nonnarcotic analgesic. The alternative, utilizing as needed medications would introduce too much background noise depending on the threshold of the patient, parent, nurse, and house staff for demanding medications. The PCA bypasses these thresholds and allows to detect difference in demands and delivered, in which we did not see a difference.

A possible reason that we did not see a significant difference in the time off the PCA and decrease in narcotic requirements is because the children were having minimal or low pain throughout their stay. The mean pain scores during the first few hospital days were never higher than 3 , which signifies that the children in our study had very good pain control with their narcotic regimen regardless if they received IVA. A confounding factor, however, is the non-IVA group did receive by mouth or per rectum acetaminophen for 
fevers; therefore, any benefit from IV acetaminophen could have been mitigated by the fact that the non IV acetaminophen group was still receiving doses of acetaminophen.

The limitations include our narcotic regimen was managed by our anesthesia department, therefore the pain regimen and type of narcotics was based on a nonstandardized preference by each anesthesiologist. Since they were not blinded to which patients were receiving IVA, they may have been biased toward the pain regimen they recommended. Our preoperative pain regimen was not standardized, and therefore, there may have been variability in early postoperative pain management and scores that we cannot quantify. Patient were supposed to be transition off the PCA once having bowel function and tolerating diet; yet, transition to oral pain medication occurred 36 hours in the IVA group and 53 hours in the non-IVA group after starting enteral feeds and with established bowel function. This may have been due to variability in management between surgery and anesthesia which caused the delay in transition. Lastly, our study was powered to find significance with a full day difference in transitioning off the PCA. The half-day difference was not statistically significant, given that we powered our study for a one-day difference.

\section{Conclusion}

Children given IVA showed no difference in the transition time off the PCA and to oral pain medications after laparoscopic appendectomy for perforated appendicitis. Children still used the same amount of narcotics with no change in pain perceptions. Further studies are needed to evaluate the role of IV acetaminophen in opioid sparing pain regimens after abdominal surgery in children.

\section{Conflict of Interest}

None.

\section{Acknowledgment}

The authors thank Ashley Sherman, MA, for her work with analyzing the results.

\section{References}

1 Witt WP, Weiss AJ, Elixhauser A. Overview of hospital stays for children in the United States, 2012-2014. Available at: https:// www.hcup-us.ahrq.gov/reports/statbriefs/sb187-Hospital-StaysChildren-2012.jsp. Accessed December 7, 2016

2 Newman K, Ponsky T, Kittle K, et al. Appendicitis 2000: variability in practice, outcomes, and resource utilization at thirty pediatric hospitals. J Pediatr Surg 2003;38(03):372-379

3 van den Bogaard VA, Euser SM, van der Ploeg T, et al. Diagnosing perforated appendicitis in pediatric patients: a new model. J Pediatr Surg 2016;51(03):444-448

4 Ponsky TA, Huang ZJ, Kittle K, et al. Hospital- and patient-level characteristics and the risk of appendiceal rupture and negative appendectomy in children. JAMA 2004;292(16):1977-1982

5 St Peter SD, Adibe OO, Iqbal CW, et al. Irrigation versus suction alone during laparoscopic appendectomy for perforated appendicitis: a prospective randomized trial. Ann Surg 2012;256(04): 581-585
6 Alkhoury F, Burnweit C, Malvezzi L, et al. A prospective study of safety and satisfaction with same-day discharge after laparoscopic appendectomy for acute appendicitis. J Pediatr Surg 2012; 47(02):313-316

7 Tomecka MJ, Bortsov AV, Miller NR, et al. Substantial postoperative pain is common among children undergoing laparoscopic appendectomy. Paediatr Anaesth 2012;22(02):130-135

8 Rodgers BM, Webb CJ, Stergios D, Newman BM. Patient-controlled analgesia in pediatric surgery. J Pediatr Surg 1988;23 (03):259-262

9 Cucchiaro G, Dagher C, Baujard C, Dubousset AM, Benhamou D. Side-effects of postoperative epidural analgesia in children: a randomized study comparing morphine and clonidine. Paediatr Anaesth 2003;13(04):318-323

10 Kelly LE, Rieder M, van den Anker J, et al. More codeine fatalities after tonsillectomy in North American children. Pediatrics 2012; 129(05):e1343-e1347

11 Ciszkowski C, Madadi P, Phillips MS, Lauwers AE, Koren G. Codeine, ultrarapid-metabolism genotype, and postoperative death. N Engl J Med 2009;361:827-828

12 Liu C, Ulualp SO. Outcomes of an alternating ibuprofen and acetaminophen regimen for pain relief after tonsillectomy in children. Ann Otol Rhinol Laryngol 2015;124(10):777-781

13 Kelly LE, Sommer DD, Ramakrishna J, et al. Morphine or ibuprofen for post-tonsillectomy analgesia: a randomized trial. Pediatrics 2015;135(02):307-313

14 Sutters KA, Miaskowski C, Holdridge-Zeuner D, et al. A randomized clinical trial of the efficacy of scheduled dosing of acetaminophen and hydrocodone for the management of postoperative pain in children after tonsillectomy. Clin J Pain 2010; 26(02):95-103

15 Mattos JL, Robison JG, Greenberg J, Yellon RF. Acetaminophen plus ibuprofen versus opioids for treatment of post-tonsillectomy pain in children. Int J Pediatr Otorhinolaryngol 2014;78 (10):1671-1676

16 Carney J, Finnerty O, Rauf J, Curley G, McDonnell JG, Laffey JG. Ipsilateral transversus abdominis plane block provides effective analgesia after appendectomy in children: a randomized controlled trial. Anesth Analg 2010;111(04):998-1003

17 Morton NS, O'Brien K. Analgesic efficacy of paracetamol and diclofenac in children receiving PCA morphine. $\mathrm{Br} \mathrm{J}$ Anaesth 1999;82(05):715-717

18 Liu Y, Seipel C, Lopez ME, et al. A retrospective study of multimodal analgesic treatment after laparoscopic appendectomy in children. Paediatr Anaesth 2013;23(12):1187-1192

19 Chou R, Gordon DB, de Leon-Casasola OA, et al. Management of postoperative pain: a clinical practice guideline from the American Pain Society, the American Society of Regional Anesthesia and Pain Medicine, and the American Society of Anesthesiologists' Committee on Regional Anesthesia, Executive Committee, and Administrative Council. J Pain 2016;17 (02):131-157

20 Curatolo M. Regional anesthesia in pain management. Curr Opin Anaesthesiol 2016;29(05):614-619

21 Rudd RA, Aleshire N, Zibbell JE, Gladden RM. Increases in drug and opioid overdose deaths-United States, 2000-2014. Morb Mortal Wkly Rep 2016;64(50-51):1378-1382

22 Pettersson PH, Jakobsson J, Owall A. Intravenous acetaminophen reduced the use of opioids compared with oral administration after coronary artery bypass grafting. J Cardiothorac Vasc Anesth 2005;19(03):306-309

23 El Chaar M, Stoltzfus J, Claros L, Wasylik T. IV acetaminophen results in lower hospital costs and emergency room visits following bariatric surgery: a double-blind, prospective, randomized trial in a single accredited bariatric center. J Gastrointest Surg 2016;20(04):715-724

24 McNicol ED, Ferguson MC, Haroutounian S, Carr DB, Schumann R. Single dose intravenous paracetamol or intravenous propacetamol 
for postoperative pain. Cochrane Database Syst Rev 2016;(05): CD007126

25 Wong I, St John-Green C, Walker SM. Opioid-sparing effects of perioperative paracetamol and nonsteroidal anti-inflammatory drugs (NSAIDs) in children. Paediatr Anaesth 2013;23(06):475-495

26 Ohlsson A, Shah PS. Paracetamol (acetaminophen) for prevention or treatment of pain in newborns. Cochrane Database Syst Rev 2015;(06):CD011219

27 Machoki MS, Millar AJ, Albetyn H, Cox SG, Thomas J, Numanoglu A. Local anesthetic wound infusion versus standard analgesia in paediatric post-operative pain control. Pediatr Surg Int 2015;31 (11):1087-1097

28 Ousley R, Burgoyne LL, Crowley NR, Teague WJ, Costi D. An audit of patient-controlled analgesia after appendicectomy in children. Paediatr Anaesth 2016;26(10):1002-1009

29 Thanapal MR, Tata MD, Tan AJ, et al. Pre-emptive intraperitoneal local anaesthesia: an effective method in immediate post-operative pain management and metabolic stress response in laparoscopic appendicectomy, a randomized, double-blinded, placebocontrolled study. ANZ J Surg 2014;84(1-2):47-51 